\title{
Vigilando las fronteras mediante dispositivos tecnológicos de control de las personas, sus cuerpos y sus hábitos ${ }^{1}$
}

\author{
Dra. Ana M. González Ramos
}

Envío recibido 2020-05-05 aprobado el 2020-12-19

Cómo citar este artículo:

González Ramos, A. M. (2020). Vigilando las fronteras mediante dispositivos tecnológicos de control de las personas, sus cuerpos y sus hábitos. Trans-Pasando Fronteras, (16). https://doi.org/10.18046/retf.i16.3928

\begin{abstract}
$1 \quad$ La presente investigación ha sido llevada a cabo gracias a los proyectos de investigación GENTIC, Relaciones entre el género y las TIC en la Economía del Conocimiento (2014SGR1199), Flujos de los recursos humanos en Cienciay Tecnología (CSO2009-09003), Study on ICT to support everyday life integration of immigrants or ethnic minority, (IPTS-2011-J04-02-RC) y Contribución en el conocimiento científico entre el personal de investigación en formación (EA2006-0099). Una versión anterior de este manuscrito ha sido defendida en el VIII Congreso de la Sociedad Académica de Filosofía. En relación con ello, agradezco a la catedrática M. José Guerra Palmero su invitación a participar en la mesa "Éxodos. Migraciones, Tránsitos y Retornos" y a todas las personas asistentes por sus sugerentes comentarios.
\end{abstract}

2 Profesora de la Univesidad Autónoma de Barcelona 


\section{Resumen}

La tecnología forma parte de los actuales sistemas de control y vigilancia, atraviesa las políticas de seguridad de los estados, y modelan las rutinas y los hábitos de las personas que cruzan las fronteras. Este trabajo reflexiona sobre el papel de las tecnologías contemporáneas en las disciplinas de las personas viajeras, cómo se cruza con los derechos de movimiento y transporte atendiendo a diversas circunstancias (estatus legal, económico, género) de las personas que se desplazan de un país a otro. La finalidad de este trabajo es demostrar que los dispositivos de control y vigilancia transfronterizos, si bien necesarios, deberían ser instrumentalizados para garantizar el bienestar de la ciudadanía y el respeto a los derechos humanos.

Palabras clave: tecnologías de la disciplina, seguridad, afectos, derechos humanos, fronteras 


\title{
Guarding the borders through technological devices to control people, their bodies and their habits
}

\begin{abstract}
The technology is part of the current systems of control and surveillance, intersects the states security policies, and shapes routines and habits of people crossing borders. This work address on the role of contemporary technologies in the travellers' discipline, how it intersects with the mobility and transport rights taking into account several circumstances (gender, legal, economic status) of people who move from one country to another. This work aims to show that cross-border control and surveillance devices are necessary, but they should be instrumentalized for pursuing the well-being of citizens and the respect of human rights.
\end{abstract}

Keywords: discipline technologies, security, affects, human rights, borders 


\section{Introducción}

\section{Domesticidad de los hábitos mediante las tecnologías, organización social y desarrollo}

Las tecnologías dominan distintas esferas de nuestras vidas, así como de la organización sociopolítica contemporánea, moldeando nuestras costumbres y hasta nuestra corporalidad. Desde que Foucault (2002 [1975]) vinculó la tecnología con la gestión del poder y la docilidad de los cuerpos, esta relación ha influido decisivamente en el campo científico y filosófico. Según este autor, las distintas organizaciones sociales utilizan dispositivos tecnológicos específicos con los cuales es posible articular el orden social, los hábitos y las costumbres de los miembros de una sociedad. Podemos poner un ejemplo con el dispositivo tecnológico más característico de nuestra época: los móviles. Los "teléfonos inteligentes" regulan nuestros hábitos desde que despertamos, ofreciéndonos los buenos días, comunicándonos la temperatura exterior - por tanto, sugiriéndonos una manera de vestir -, durante el día, avisándonos cuál es nuestra próxima tarea, si las personas de nuestra agenda electrónica están disponibles - por tanto, estableciendo un control sobre las personas que nos importan -, hasta que nos vamos a la cama, informando a nuestros contactos que hemos desconectado el dispositivo electrónico.

No es un fenómeno nuevo, las tecnologías han delineado las rutinas sociales de los seres humanos desde los albores de la humanidad. Las tecnologías características de sociedades cazadoras y recolectoras seguramente impusieron rutinas concretas relacionadas con la construcción, utilización y reparación de sus herramientas. Los grandes inventos tecnológicos, la rueda, el ferrocarril, la imprenta, la electricidad, facilitaron organizaciones económicas, políticas y sociales diferentes a la de épocas pasadas. En definitiva, como explican autores de los Ilamados estudios STS - Science, Technology and Society -, las tecnologías ocupan un lugar primordial en la configuración de las vidas 
de las personas (Bijker, Hughes y Pinch, 1986; Blázquez Graf y Flores, 2005; Akrih, 2006).

Su influencia en la cotidianidad de la ciudadanía ha venido a llamar a este proceso de penetración de las tecnologías y de apropiación de las tecnologías como una "domesticación" de las tecnologías (Lie y Sorensøn, 1996). La tecnología se ha asociado al desarrollo humano, innovaciones adoptadas a lo largo de la historia para mejorar nuestras oportunidades como especie (Hughes, 1987; Cooke y Leydesdorff, 2006). La destreza para construir y emplear tecnología sofisticada es considerada la ventaja competitiva de los seres humanos frente a las competencias evolutivas atribuidas a las otras especies (de la inespecífica ventaja del "mono desnudo" pasando por el llamado homo habilis y el hombre racional, científico y tecnológico denominado homo sapiens sapiens). La tecnología se erige como uno de los pilares fundacionales de la civilización y la humanidad siente una confianza ciega por las tecnologías (Chun, 2006).

Los ejemplos precedentes aluden al papel de las tecnologías en la civilización humana, pero qué papel juegan en la vida contemporánea las tecnologías de la información y la comunicación y, en particular, en el ámbito de la seguridad y vigilancia de las fronteras. Las naciones siempre han procurado adoptar los dispositivos de control más sofisticados posible para controlar su territorio. En este trabajo sostengo que, en la actualidad, no solo atañe a la frontera en sí misma sino a todos los actos relacionados con la movilidad de las personas nacionales y extranjeras. Las personas viajeras cuentan con una disciplina basada en los protocolos de seguridad, que están modelados por las tecnologías avanzadas de vigilancia de los movimientos de todo tipo de personas y mercancías.

Este artículo está conformado por cinco apartados de acuerdo al siguiente esquema. Tras la presente introducción, la siguiente sección analizará las normas sociales en relación a la seguridad transfronteriza 
y la cesión de derechos individuales en favor de la confianza (o la ilusión) de obtener seguridad, eje teórico que sostiene este trabajo. A partir de este punto, se analizará el impacto de la gestión tecnológica en las fronteras para la población inmigrante (población excluida y carente de los derechos de las sociedades de recepción), los actores que intervienen y la naturaleza de la supervisión y control transfronteriza. El siguiente apartado abordará el impacto de las medidas de control tecnológico en las fronteras para la ciudadanía global, para analizar cómo las tecnologías moldean y afectan a sus cuerpos, identidades y voluntades, diferenciando impactos de género. Finalmente, las conclusiones sintetizan los puntos principales y reflexionan sobre las consecuencias de aplicar un control de las fronteras tecnológico en su capacidad de distribuir los derechos de ciudadanía sin una perspectiva ética, sino basada en el control y la seguridad.

\section{Desplazamientos y dispositivos tecnológicos: normas, hábitos y cesión de derechos}

El aumento de los flujos de población es mucho mayor en la actualidad que en cualquier época anterior (Castles y Miller, 1993; Sassen, 2007), lo cual supone un reto para la ciudadanía y para los estados. Los desplazamientos se relacionan con múltiples objetivos, profesionales (científicos, expatriados, inmigrantes cualificados...), de ocio (turistas, viajeros, excursionistas, deportistas...), políticos (desplazados, refugiados, exiliados, activistas). Los desplazamientos transfronterizos se han materializado para el conjunto de la población mundial, con un mayor número de desplazamientos protagonizados por personas de cualquier edad, condición física, capacidad económica o social, puesto que la riqueza solo mejora la calidad del transporte y las comodidades (y la falta de recursos no impide el desplazamiento solo dificulta y precariza el viaje).

Las tecnologías permiten al mismo tiempo el transporte y el control del flujo de mercancías y personas. Las fronteras están equipadas con 
dispositivos de control y vigilancia para gestionar el paso ordenado de las personasy, portanto, de sus identidades, sus cuerpos y las mercancías que portan. La identidad de los viajantes se supervisa mediante el pasaporte o el título de traslado, lo cual remite al concepto de ciudadanía: ¿quién es considerado ciudadano y de qué estatus disfruta, el cual le otorga un derecho de circulación? Los cuerpos de las personas son inspeccionados, nos aseguramos de que la vestimenta no esconda objetos peligrosos o prohibidos por la normativa de viajes. Se examinan los códigos de vestimenta y la etiqueta personal, comportamientos sospechosos (como, por ejemplo, si parece borracha, drogada o enferma) serán detectados y censurados pudiendo incluso quedar en suspenso su derecho a realizar viaje temporalmente. Finalmente, pero no menos importante, se examinan las propiedades con las que se viaja: lo cual está relacionado con los mercados, los impuestos y aduanas, y también con qué tipo de artículos están permitidos o prohibidos durante el viaje.

Por tanto, los dispositivos tecnológicos de gestión de las fronteras son usados para domesticar nuestros hábitos y costumbres, incluso llegandoa configurar un "ritual específico de viaje"; un catálogo de hábitos que están permitidos y otros que están prohibidos que cumplimos a rajatabla para asegurarnos un viaje feliz. Así, por ejemplo, hemos adoptado el hábito de acercarnos al aeropuerto al menos una hora antes de la salida del vuelo. Esa inversión de tiempo está asociada con el cumplimiento obligatorio de unas medidas de seguridad que, sin embargo, son innecesarias cuando realizamos el mismo trayecto en un tren de alta velocidad. El aeropuerto es nuestro constructo tecnológico más sofisticado y ha creado normas indiscutibles: que hagamos al menos dos colas (la de seguridad del aeropuerto y la de seguridad de la nave), que mostremos nuestro pasaje en un primer punto y el pasaporte en un segundo lugar. Aunque ¿por qué en diferentes aeropuertos nos sometemos a normas distintas, diferentes tipos de vigilancia y control? Aunque los protocolos están unificados desde las agencias internacionales de transporte, cada país es soberano para determinar su propia reglamentación y la aplicación es diferente en cada aeropuerto. 
Durante el paso transfronterizo cedemos parte de nuestra autonomía, construyendo unaeconomía de los afectos resultante de la aplicación de las normas que han sido determinadas por las autoridades supraindividuales, que velan por nuestra seguridad. Sarah Ahmed (2010: 35) ha afirmado que "la asociación entre los objetos y los afectos se conserva a través del hábito", el traspaso de las fronteras tiene rituales de seguridad que mueve afectos, configura comportamientos y delimita nuestra voluntad. Separar los aparatos electrónicos (llevarlos encendidos o apagados), sacar las monedas, los efectos personales en bolsas trasparentes y hasta descalzarse se justifican por seguridad contra ataques terroristas $u$ otros actos criminales. Nos desprendernos de los líquidos y productos de higiene personal en contenedores disponibles para ello antes del paso de seguridad cumpliendo una normativa que se corresponde con los impuestos y los mercados.

Chun (2006) ha advertido que el sentimiento de libertad no ha desaparecidoapesardelasupervisióncadavezmássutilypotenteejercido mediante dispositivos tecnológicos. La ciudadanía sigue confiando en su capacidad de agencia, a pesar del deterioro de su capacidad de acción. El control es ahora invisible (p. e., información personal en las bases datos, redes de dispositivos y cámaras de seguridad electrónicas, etc.), lo cual lo hace más aceptable o quizá aún no haya sido suficientemente discutido. El omnipresente control de las tecnologías de la información $y$, muy especialmente de nuestra imagen, se fundamenta en el sentido de vulnerabilidad; las tecnologías de la vigilancia por imágenes proporcionan una ilusión de invulnerabilidad, de control y seguridad (Lyon, 2004; Chun 2006). Sin embargo, la época contemporánea es tan fluida y llena de incertidumbres que todo es inseguridad; la seguridad es imposible de alcanzar actualmente (Beck, 2008).

Esta ilusión de control atribuido a las tecnologías avanzadas de seguridad sería impensable sin la construcción de un entramado de hábitos y códigos que rodean los momentos y los espacios donde se desarrollan los viajes, que modelan nuestros afectos hasta convertirlosen 
una intromisión de la intimidad personal. Las normas y rutinas asociadas a las tecnologías en las fronteras cumplen una función similar a los sistemas rituales de cualquier comunidad antropológica: si la tecnología es el tótem proveedor de seguridad para la ciudadanía, los rituales son necesarios para confiar en su magia, es el pegamento que sostiene el apego de todos los individuos alrededor de los dispositivos de protección. Los protocolos de seguridad guían nuestros movimientos constantemente, desde la puerta de entrada hasta nuestro destino final, y, durante el trayecto, nuestra voluntad queda temporalmente en suspenso. Esta articulación de la vida social pone de manifiesto la docilidad de nuestros cuerpos, de nuestros comportamientos normativizados (Foucault, 2002).

La repeticiónyel protocolo neutralizan la individualidad, nos imponen un hábito que va más allá de nuestro sentido común, reconfigura nuestros afectos e incluso nuestra capacidad crítica ${ }^{2}$. Pareciera que las relaciones humanas se han minimizado en favor del control y la seguridad, en los pasos fronterizos sólo queda el frío protocolo de actuación. En las zonas de paso nos ponemos en cola, solo hablamos con la persona anexa para animarla a ir más deprisa, preferimos que los agentes de seguridad no nos hablen pues lo contrario significa que han detectado algún problema con nuestro equipaje o zapatos, etc. y, su papel es repetir movimientos, emitir siempre el mismo mensaje de seguridad como si fuera una grabación. En algunos lugares, se dispone de máquinas con tres emoticonos que permiten valorar el servicio recibido: sistemas de reputación social que sustituyen a la amabilidad y al saludo, el agradecimiento y las fórmulas de despedida.

\section{Vigilancia tecnológica en las fronteras dirigida a las personas "sin derechos"}

Según Grabitz (1992) las fronteras son una manifestación física

2 El seguimiento de un protocolo sofisticado y sin sentido, que pone en suspenso la individualidad y la razón, ha sido descrito con brillantez en obras como El Proceso (Kafka, 1925) y El Castillo (Kafka, 1926). 
de la soberanía de los estados, que otorga a sus responsables la discrecionalidad de permitir o prohibir la entrada de personas u objetos. Sin embargo, qué significa "soberanía" en un mundo globalizado, con límites transfronterizos que gestionan ciertos aspectos fundamentales de la economía, la sociedad, la seguridad y la salud de todos o de un grupo de países. La globalización y la conciencia de estar en un mundo transfronterizo son ideas centrales de la época contemporánea (Urry, 2007), que nos enorgullece como integrantes de las sociedades avanzadas. Sin embargo, en vez de crear una identidad transnacional que permita la libre circulación de las personas y garantice el respeto a los derechos humanos, se han establecido reglas de entrada, salida y permanencia en cada estadonación o grupo de países, por ejemplo, Mercosur, Schengen... (Sassen, 2007; Nussbaum, 2007; Alvites Baiadera, 2019).

Enel ámbito de las migraciones, las tecnologías son empleadas tanto para la movilidad de los flujos de personas como para la regulación de sus actividades. Las tecnologías separan a las personas entre sí, asignándoles derechos (inmaterialidad) y regulando el transporte de elementos físicos (materialidad), se les asigna un lugar espacio concreto y se los distribuye en categorías (legales, ilegales, nacionales, extranjeros, etc.). Cada persona queda encapsulada dentro de un marco fronterizo (un territorio, un estatus legal a partir del cual, se les atribuye una serie de derechos y el reconocimiento de una serie de recursos). Así, por ejemplo, los menores extranjeros no acompañados son sometidos a procesos de identificación utilizando dispositivos tecnológicos, una radiografía que determina si son adolescentes o adultos (Abbing, 2011); siguiendo una norma jurídica que ignora los diferentes significados culturales que tiene el término niño o adulto en los diversos países (particularmente, en las comunidades de procedencia de esas personas). El estatus legal de las personas es encapsulado en cada frontera nacional, establecida por una medición tecnológica. De esta manera, los derechos de una misma persona se configuran de manera diferente a cada lado de la frontera. 
En los límites de las fronteras queda la criminalidad, la violencia y la muerte. Así, en ciudades fronterizas como Ceuta y Melilla en España, Juárez y El Paso en México, la ley se establece por parte de grupos organizados, cuyo papel parece ser únicamente el mantenimiento perpetuo de la inseguridad. Y, a su vez, esto hace que los espacios fronterizos sean utilizados por la ciudadanía con derechos como un reflejo antagónico de la situación que tratan de evitar. La ciudadanía con derechos teme que los delitos y el caos se extiendan inexplicablemente hasta su propia casa. En este sentido, la utilización de cualquier medio de represión y de alejamiento de las personas expulsadas queda justificada moralmente. Se estipulan lugares para recluirlas, como la Isla Stege Bay en Lindholm, al sur de Dinamarca, donde se pretende instalar a los inmigrantes que cometan algún delito.

La alarma social legitima la cesión de derechos activando nuestros afectos: sentimientos de rechazo, temor frente a los otros y miedo a la posibilidad de ser sus víctimas. No importa lo remota e improbable que sea la amenaza. La expresión de los otros, con hábitos y vestimenta diferentes, sujetos racializados y disminuidos en su humanidad, justifican la transgresión de derechos humanos, porque sentimos que estamos en una situación de amenaza permanente de nuestro modo de vida. La función de las fronteras es determinar quién está de un lado o del otro: si se forma parte de la población amenazada o la potencialmente peligrosa; ¿qué derechos se tienen frente a la ciudadanía con plenos derechos? ¿qué derechos individuales ceden los ciudadanos con derechos para combatir el sentimiento de inseguridad que les atenaza? ¿qué nivel de vulnerabilidad existe precisamente por no formar parte de la población con derechos?

Las vallas, las cámaras fijas dirigidas a objetivos humanos, los globos aerostáticos, etc. establecen una vigilancia constante. El control tecnológico de las fronteras se plantea de manera aséptica, perdiendo de vista las consecuencias que tiene para los sujetos y la interferencia sobre aspectos fundamentales de los derechos humanos. Como en la 
guerra, la tecnología de gestión de fronteras ha evolucionado hacía un control a distancia, con la despersonalización o mínimo contacto humano, lo que convierte a las personas en no-humanos, objetivos políticos y de seguridad sin derechos. Los dispositivos de control biométricos y de escáner utilizados en las entradas y salidas de los puertos y aeropuertos proporcionan una cortina de protección para los humanos (los ciudadanos con derechos) respecto a los otros (los expulsados/sin derechos). Las tecnologías separan a quienes tiene derechos y quienes quedan excluidos (los emigrantes ilegales o quienes son sospechosos de la comisión de algún delito).

La conformidad, más o menos mayoritaria de la población, sobre este estado de cosas proviene de la idea seductora de que existe una zona de bienestar y seguridad (Lyon, 1994; Goldsmith y Crawford, 2014). La ciudadanía ha tomado conciencia de la situación mediante un conjunto de pruebas (noticias y sucesos leídas o sentidas en los medios de comunicación) que provocan alarma social y que "demuestran" la inseguridad que reina en nuestras ciudades. Los medios de comunicación, los gobiernos y los especialistas refuerzan este sentimiento, creando categorías como zonas de riesgo y lugares donde es necesario vigilar y controlar nuestras pertenencias. Cuanto mayor es la alarma social, mayor es el consenso respecto a la necesidad de poner en marcha una acción colectiva defensiva (Chun, 2006; Lunt y Livingstone, 2010; Levmore y Nussbaum, 2010).

La amenaza es tan alta que el estado es incapaz de asegurarla por sí solo (Lessig, 1999; Chun, 2006; Weiss, 2014); no solo se coordina con otros estados y agencias gubernamentales, sino ahora también con entidades extragubernamentales y con empresas privadas de seguridad. Linda Weiss (2014) ha acuñado el término national security state para aludir a las agencias militares y policiales, que junto a las agencias de energía y de política científica (en Estados Unidos, la autora cita, por ejemplo, la NSF, National Science Fundation, y el National Institute of Health) que controlan la vida cotidiana de la ciudadanía. Según la autora, estas agencias se inmiscuyen en ámbitos 
de actuación tradicionalmente gubernamentales, interviniendo incluso en asuntos políticos, militares y policiales, desdibujando así lo militar y lo civil, así como la naturaleza pública y privada de los recursos de seguridad de un país.

Así pues, la gestión tecnológica se complejiza y sofistica con la incorporación de otros actores. Agencias como FRONTEX, la Agencia Europea para la Gestión Operativa de Cooperación en las fronteras externas cuya finalidad es:

"coordinar la cooperación operativa entre los estados miembros en la gestión de las fronteras externas, asistiendo el entrenamiento de los guardas nacionales fronterizos, incluyendo el establecimiento de estándares de entrenamiento comunes, la ejecución de análisis de riesgos, el seguimiento de las investigaciones en desarrollo relevantes para el control y supervisión de las fronteras exteriores, la asistencia a los estados miembros en circunstancias de requerimiento técnico y operacional en las fronteras externas, y la provisión a los estados miembros del apoyo necesario en la organización de operaciones de retorno conjuntas".

En su catálogo de buenas prácticas, (FRONTEX, 2015) especifica que cuenta con el Ilamado diseño ABC (Automated Border Control), considerado indispensable y no exclusivo para controlar las fronteras, el cual está compuesto por: una o dos barreras físicas (puertas simples o dobles, e-Gates), equipamientos de monitorización y control de estación para el operador, documento de lectura (aparatos ópticos incluyendo radio-frecuencia modular de lectura), varios aparatos de captura biométrica (cámeras y/o lectura de huellas digitales), interfarces de usuario (monitores, señales LED, aparatos de audio, botón de pánico), unidades de procesamiento y aparatos en red (PC, controladores, hubs), cámaras y sensores para la vigilancia (CCTV, detección de puerta trasera y detección de equipaje).

En su análisis respecto a las recientes campañas de Frontex, Rodier (2010) determina que es un arma prêt-à-porter contra un enemigo situado en las puertas, dejando atrás el compromiso de asilo acordado 
en la UE. La instauración del miedo respecto a posibles ataques violentos de grupos organizados, llamados terroristas, justifica la necesidad de protección de las fronteras y un modo de operar represivo y contundente. El miedo actúa como un disolvente social, que deshace la razón crítica y diluye los derechos políticos y sociales de la ciudadanía (Lyon, 1994; Ditton, 2000; Chun, 2006).

Las empresas privadas también se equipan para intervenir en el control de las fronteras. Por ejemplo, el grupo empresarial tecnológico GMV, fundado en 1984, de ámbito internacional cuenta con productos y servicios de mercado (es decir, abierto a la compra directa del público y no exclusivamente a los estados) dirigidos a "apoyar los procesos de vigilancia de sus clientes a través de soluciones tecnológicamente avanzadas". En su catálogo de productos ofrece servicios de consultoría e ingeniería, desarrollo de software y hardware relacionado con sistemas de mando y control C4ISTAR, procesado de datos y señales, sistemas de inteligencia, ciberdefensa, simulación, aplicaciones militares del espacio, aplicaciones GPS, EGNOS y Galileo, sistemas integrados de control de accesos, presencia, vigilancia y seguridad, sistemas de vigilancia de fronteras, sistemas de gestión de emergencias y crisis, centros 112, sistemas de seguimiento y gestión de vehículos y personal de fuerzas de seguridad. GMV dispone de una larga y diversa lista de recursos tecnológicos disponibles para entidades públicas y personas privadas, cuya finalidad es mantener seguras las vidas de las personas confiables, con estatus legal, y alejarlas de la amenaza de peligro que suponen las otras.

Por tanto, en las fronteras, la población desplazada (la legal y la "ilegal") debe acceder a las zonas de seguridad mediante la superación de las barreras tecnológicas gestionadas por los estados y las agencias supraestatales y empresas privadas, que ejercen labores conjuntas de supervisión y vigilancia tanto física como a distancia. 
La particularidad de la tecnología de control de fronteras más avanzada es que ya no ejerce solo una vigilancia ostensible, sino que se trata de ejercer un control disuasorio, llevado a cabo por dispositivos más discretos e invisibles (asociados a la infalibilidad) como los ojos electrónicos y las grandes bases de datos conectadas. La imagen de los guardas en sus postas ha sido prácticamente relegada a una cuestión simbólica, pues otros tipos de dispositivos de vigilancia tecnológicamente más avanzados, sofisticados, menos visibles y, por tanto, sutiles distribuye a las personas entre los pasos fronterizos.

En resumen, la tecnología configura los bordes situados entre dos espacios desiguales en derechos y oportunidades; la idea de frontera se erige como un límite para las libertades y el bienestar de las personas. Un concepto que históricamente ha estado ligado a la guerra y a la disputa entre los pueblos, se ha convertido en el confín de nuestros deseos y subjetividades, del disfrute de derechos o de la exclusión de los sin derechos. Las fronteras representan los límites del miedo (la vulnerabilidad de la humanidad) y la ilusión de seguridad. Las tecnologías supervisan y controlan la gestión de esos derechos (clasificando a las personas, permitiéndoles acceso o no, proporcionándoles un visado de residencia o una orden de expulsión), determinando sus rutinas (establecidas para acompañar y significar las medidas de seguridad impuestas por la tecnología), y moldeando sus afectos y voluntades. Los estados y las agencias supraestatales o privadas también juegan un papel fundamental en la gestión del paso y de los derechos de la ciudadanía, cada vez con un mayor desarrollo de tecnologías avanzadas, que se ejercen desde la distancia geográfica y la asepsia social representada por las máquinas.

\section{Control de la materialidad e inmaterialidad en las fronteras}

En las fronteras se controla la materialidad (mercancías, cuerpos) y la inmaterialidad (derechos, recursos, acogida o expulsión) mediante el establecimiento de barreras y permisos. La supervisión de la 
materialidad se refiere al control de las mercancías (portadas o enviadas) y del cuerpo, es decir, de los códigos de vestimenta y el comportamiento de las personas viajeras. La supervisión de la inmaterialidad concierne a la identidad del pasajero y a los derechos y recursos que de ella se derivan (a través del pasaporte, el boleto o billete, la aparición en las bases de datos de seguridad internacionales...). A pesar de la objetividad que pretenden validar las tecnologías avanzadas de seguridad trasfronterizas, los juicios subjetivos y los prejuicios sociales desbordan esa asepsia. Las personas racializadas, vestidas modestamente son tratadas de manera diferente (ellas y sus pertenencias) en las fronteras.

Respecto a la materialidad del cuerpo, por ejemplo, en los aeropuertos, se han establecido protocolos específicos para quienes portan prendas religiosas contrarias a los usos normativizados. Las mujeres con pañuelo islámico y los hombres con turbantes sij tienen derecho a pasar con estas prendas siempre que no se eleve la alarma de metales o, también, pueden ser inspeccionados mediante detectores de metales portátiles y solo en caso de duda se les puede pedir que se despojen de la prenda en una zona privada. Estas medidas excepcionales han sido tomadas tras la reclamación de dichos colectivos en defensa de sus derechos civiles y religiosos. En cambio, sigue conservándose la reglamentación relativa al uso del calzado, especialmente relacionado con el uso de tacones, botas o calzado femenino, a pesar de la medida se originó en 2001 cuando Richard Reid intentó camuflar una bomba en el tacón de su zapato masculino. Por tanto, solo un juicio subjetivo asocia un tipo de zapatos u otros a una amenaza de seguridad.

Las reglas de vestimenta y colocación de artículos en el equipaje también están justificadas por razones de seguridad. Viajar con ropa cómoda está igualmente sustentada en la libertad de movimientos si surgiese una emergencia; y, distribuir los artículos en el equipaje de mano y maletas de mayor volumen, que se dejan en la bodega o compartimentos menos accesibles, con la necesidad de llevar algunos artículos imprescindibles en la cabina, asiento del autobús, litera o 
camarote. Para la ciudadanía que lleva a cabo desplazamientos con cierta frecuencia, preparar un viaje supone un adiestramiento (y un modelado) de sus rutinas. Su protocolo de viaje está conformado por las costumbres interiorizadas a través de su experiencia de movilidad geográfica, mayor cuanto más frecuentemente realice un mismo tipo de viajes por tierra, mar o aire.

Una vez en la frontera, en el control de seguridad, los agentes y los dispositivos tecnológicos supervisan nuestra materialidad (cuerpo y equipaje) e inmaterialidad (identidad, estatus de legalidad, comportamientos) de manera sistemática y sin provocar resistencia en ningún momento. Incluso aunque la supervisión parezca arbitraria o discriminatoria, las personas que viajan se someten al escrutinio y al exacto cumplimiento de las normas. De hecho, se hace exhibición de un comportamiento dócil, que refuerza la impresión de orden y seguridad (con el que se quiere convencer especialmente a los agentes de seguridad), y que evidencia un momento de suspensión de nuestra voluntad (la suspensión de nuestra individualidad y descorporalización). Cuantos más obstáculos se prevean (si nuestro visado está a punto de caducar, si nuestro aspecto físico racializado Ilama la atención, etc.), más docilidad se exhibirá tratando de evitar la atención suspicaz de los agentes.

Respecto al control de la materialidad, recientemente, se han popularizado algunos reality shows, como, por ejemplo, en el canal Discovery Max, donde se muestran el traspaso de fronteras de personas o de mercancías. En estos programas se exhiben casos reales, grabaciones realizadas en las fronteras y en las aduanas, con la intención de mostrar aquellos casos sospechosos, cómo actúan las fuerzas de seguridad y las personas implicadas. La masificación del mensaje en los medios de comunicación cumple una función auto-justificativa y propogandística, según Jodi Dean (2007), para establecer las fuentes de poder y los derechos de la ciudadanía. Los programas son un programa ligero para entretener a la audiencia que, al mismo tiempo, cumplen 
la misión de trasmitir un cierto tipo de advertencia: "no lo hagas, no lo intentes, lo descubriremos". En la filmación de estos casos, la intimidad de las personas queda expuesta. Las infracciones al empaquetar medicamentos o comidas fundamentales para ellas personas son tildadas de sospechosas. Muchas veces, son artículos esenciales en la cultura de las personas que las portan, pero que no están permitidas en el país de destino (un medicamente a base de animales disecados o un alimento considerado contrabando). Sus equipajes y artículos ocupan el primer plano, mientras que las personas pasan a un segundo plano, o cuando aparecen se difuminan sus rostros. Pero, ocultar sus caras no es respetar su intimidad, pues se muestran sus actos, el contexto donde se producen (la frontera terrestre con Canadá, en el aeropuerto de Miami, etc.), así como otros elementos identificativos y, sobre todo, privados (vuelve de Afganistán tras haber acudido al entierro de su madre, cogió el coche de su expareja sin permiso para acudir a la comida familiar de Acción de Gracias, etc.). Y, en todos los casos, se muestra la malicia, la torpeza o la conducta delictiva, que estigmatiza a esas personas.

Dos características Ilaman la atención sobre estos programas. En primer lugar, las rutinas de las que se recela antes de examinar la mercancía; las señales que alertan a los agentes fronterizos, según se describe por la voz en off, el nerviosismo, los comportamientos erráticos, las trayectorias de viaje sospechosas, el aspecto sospechoso, las atribuciones étnicas y culturales. En segundo lugar, la participación de la tecnología como criterio definitivo para desvelar el delito. La sospecha de los agentes debe ser avalada por la tecnología: se pasa el equipaje o la ropa por los escáneres, se realiza una prueba de detección de productos químicos, etc. El juicio certero no se aplica a los profesionales entrenados para detectar un delito sino a las tecnologías (Ditton, 2000). Los protocolos de seguridad tecnológicos son exhibidos como elementos objetivos que permiten identificar a los infractores de la ley, para tranquilidad del resto de la ciudadanía incluidos los propios expertos que se apoyan en una información extraída mecánicamente. 
La prueba tecnológica se muestra como la garantía de superación de la arbitrariedad, la subjetividad o los posibles juicios erróneos que pudieran cometer los agentes de seguridad.

En cuanto al control de la inmaterialidad, las costumbres fijadas a partir de los protocolos de seguridad se han convertido en el cemento social de nuestro sistema (Elster, 2005). Los elementos que establecen el derecho de paso van más allá de la lógica y la razón crítica. ¿Cómo si no puede confiarse en la sinceridad de las respuestas de las tarjetas de inmigración? Por ejemplo, el formulario ESTA -Electronic System for Travel Authorization- de Estados Unidos pregunta: "si se ha estado o está involucrado en alguna actividad de espionaje o sabotaje, en actividades terroristas o de genocidio" ¿alguien puede pensar que esas preguntas están sujetas a una exigencia de veracidad? ¿se pretende obtener una respuesta afirmativa cuando el sujeto tiene la intención de cometer esos delitos? Esas respuestas no producen veracidad o falsedad, se emplean como justificación del delito de falsedad cometido, cuando ya se ha cometido y se tienen evidencias, en cambio, crean ilusión de invulnerabilidad.

Los hábitos y rutinas de seguridad, construidos a partir de los dispositivos de vigilancia electrónica, están relacionados con la fundamentación y sostenimiento de un poder sutil e invisible que pretende garantizar la seguridad ciudadana (Ditton, 2000; Chun, 2006). La consciencia de esa omnipresencia del poder tecnológico merma la capacidad de escrutinioy juicio crítico. Los hechos absurdos, contrarios a nuestros valores y principios emergen solo en ocasiones excepcionales: cuando una familia es obligada a bajar del avión debido a la "conducta extraña" de una niña de 15 años autista (mayo 2015) o cuando un viajero es expulsado por la fuerza de un avión por 'overbooking' (abril 2017). La poderosa representación de las tecnologías y los estrictos protocolos de control y seguridad modelan nuestros hábitos y afectan a la capacidad de agencia. Nuestra capacidad crítica se desactiva, la mayoría de las veces, ni siquiera nos planteamos rebelarnos; por el 
contrario, normalmente, manifestamos rutinas descorporalizadas y desconcienciadas siguiendo parámetros conocidos y establecidos por una autoridad supraindividual que dicta los códigos de conducta (los que están permitidos y los que están prohibidos). La aceptación de esas normas se sustenta sobre nuestras emociones, provocadas por los sentimientos de vulnerabilidad, temor, seguridad, etc.

Todas nuestras acciones, incluso deseos, están filtradas por la regulación del sentimiento de seguridad. La industria área es la más absurda: durante el vuelo no se puede fumar, puede encenderse los dispositivos electrónicos pero no durante el aterrizaje y despegue, también, dependiendo de la compañía y tamaño del aparato electrónico. Docilidad adoctrinada por la norma establecida en los derechos de los pasajeros (marítimo, terrestre o aéreo), en la información reflejada en los títulos de transporte, en los consejos dictados por voces anónimas mediante altavoces, por los cambios normativos de las agencias de transportes transnacionales anunciados en los medios de comunicación.

Los hábitos y costumbres que son entrenados a base de repetición, y reforzados a través de la consecución de una experiencia satisfactoria de viaje (tranquila y sin problemas). Por ello, también miramos con cierto desdén al pasajero que actúa de una manera extraña o que manifiesta menos experiencia como viajero y que avanza más lentamente en la fila o no sabe qué hacer en el arco de seguridad. Estas personas obstaculizan la satisfacción (la velocidad de movimientos) del viajero frecuente y, en ese momento, como sostiene Sara Ahmed (2004), nuestros afectos hacia la colectividad se anulan.

La atención que recibe unas personas u otras es muy distinta dependiendo de su estatus. Cada grupo social está sometido a rituales segregados, que nos separa y diferencia conforme a los derechos y recursos de los que somos (o no) beneficiarios. También en esta cuestión intervienen los dispositivos tecnológicos. Las cámaras de televisión reciben al emigrante futbolista, una celebridad para el 
público mientras los escáneres de la aduana representan la bienvenida del emigrante que carga con una maleta pesada y sospechosa por su tamaño (¿sospechosa por sus dimensiones?). La capacidad económica ofrece confort y bienestar al viajero; por ejemplo, en el aeropuerto de Schipol las personas que viajan en clase VIP disponen de una máquina de escaneado de iris para que puedan pasar más rápida y cómodamente la zona de seguridad. La burbuja de seguridad no solo está al servicio de los derechos legales, también representan ventajas políticas y sociales para unas personas frente a las otras.

En este sentido, Agger (2003) manifiesta que las tecnologías regulan nuestras emociones, las evalúa y mercantiliza no solo al amparo de la búsqueda de seguridad, sino en el convencimiento de que se corresponden con un bienestar ligado al estatus social. La mercantilización de la seguridad, mediatizada por las tecnologías electrónicas, potencia emociones vinculadas al bienestar y al estatus económica. Así, la tecnología se pone a disposición de aquellas personas que cuentan con mayores recursos económicos para proporcionarles mayor bienestar: la fluidez del tránsito, la comodidad de la primera clase, la suspensión del escrutinio de las aduanas. Las emociones de las personas con derechos son explotadas económicamente (commodification), creando productos deseables y al alcance de ciertas personas.

\section{Vigilancia y control de los cuerpos y movimientos de las mujeres}

Donna Haraway (1988) y Sandra Harding (2004) señalan que la mirada masculina está presente en el desarrollo del conocimiento humano desde épocas históricas. Podemos encontrar la hegemonía de este enfoque androcéntrico en el diseño de las tecnológicas y en la disposición de estas en el control de las fronteras. Las tecnologías de gestión de control de las fronteras son universales sin distinción entre hombres y mujeres; por tanto, pretendidamente independiente de que la aplicación de los sistemas de seguridad tenga efectos de género diferentes. Sin 
embargo, las tecnologías electrónicas generan consecuencias diferentes, de diversa naturaleza cuando se adopta una mirada interseccional, respecto al origen, etnia, religión, identidad sexual y edad. Esta relación entre innovación tecnológica y género está ampliamente estudiada en relación a las tecnologías de la información y la comunicación. Por ejemplo, el rastreo de datos personales y el uso de big data como instrumento de análisis sin aplicar filtros sensibles a las categorías de análisis de género se considera una amenaza de especial relevancia para mujeres y niñas (Ajunwa, Crawfordy Schultz, 2017; Crawford, 2019; D’Ignazio y Klei, 2019). En el presente apartado analizaremos qué impacto ejerce las tecnologías de la vigilancia y el control para las mujeres.

No faltan evidencias reales. Los grupos organizados utilizan GPS y cámaras digitales para dar apoyo a su actividad delictiva, principales contra mujeres y niñas, empleadas en el tráfico de personas (Thakor y boyd, 2013; Oroza y Puente, 2017). También, internet se emplea como un "escaparate" global donde las mujeres se ofrecen como artículos de mercado, al gusto de quienes capacidad adquisitiva, tal y como muestra Julia Meszaros (2018) en la actividad realizada por algunas agencias matrimoniales. Las redes sociales se convierten en un dispositivo de gestión de las relaciones humanas, amplificando ciertas prácticas existentes previamente. El cyberbulling se impone como modalidad de la máxima virulencia del tradicional acoso en las escuelas, pues añade el gravamen de una mayor exposición pública (Livingstone, Stoilova y Kelly, 2016). El control social se incorpora en el diseño de las aplicaciones digitales insertando un guión de género (Akrich, 2006).

Por su parte, las tecnologías de gestión de las fronteras imponen normas y hábitos diferentes a las mujeres frente a los hombres, resultado de nuestra cultura dicotómica. Al uso de los tacones mencionado anteriormente, puede añadirse la necesidad de incluir agentes mujeres que supervisen (tienen permitido tocar) los cuerpos de las mujeres bajo los arcos de seguridad. Otro ejemplo, es que las inspecciones de seguridad relativas a la ropa femenina son más minuciosas pues parece que 
las prendas íntimas (las ballenas de sostenes, las gomas de las bragas) representan una mayor amenaza, lugares comunes donde esconder mercancías o sustancias peligrosas; al menos, así constan en los protocolos de seguridad. Las mujeres no suelen hablar de las situaciones de vulnerabilidad sufridas en los pasos fronterizos, quizá porque son consideradas habituales o, como sucede a menudo, porque la vulneración de género queda suscrita al ámbito privado y es silenciada por ser moralmente cuestionada. Sin embargo, cabe destacar la trasgresión de la intimidad que sufrimos cuando los agentes de seguridad escrudiñan nuestras ropas o al mostrar todos los artículos de uso íntimo en bolsas de plástico transparente. Una mención especial merece las máquinas de escaneado, como las del aeropuerto de Manchester, que fueron denunciadas por exponer los cuerpos de las personas que pasaban el control de seguridad. Por otra parte, las vallas fronterizas suponen un hándicap para las mujeres. Aunque pueden suponer situaciones penosas para hombres y mujeres, las experiencias negativas son muy diferentes para ellos y para ellas. La imagen que se traslada desde los medios de comunicación respecto al cruce de fronteras suele ser de migrantes ilegales hombres, raramente de mujeres. A ellas se les atribuye un rol victimizado, como "objeto de violencia" (aunque, curiosamente, también se asocian fuertemente a "sujetos de terror", por ejemplo, en el caso de las mujeres suicidas yihadistas, que llama la atención más por su rareza que porque se produzca un número de veces significativo). En definitiva, la representación más habitual de la migración está masculinizada porque se asocia a los hombres la fuerza laboral e incluso la fuerza viril necesaria para sobrellevar las dificultades de una entrada ilegal. Cuando se habla de mujeres migrantes suelen estar asociadas a sujetos-víctimas de delitos sexuales, traficadas con fines de explotación sexual. Incluso en esas ocasiones, las mujeres son mostradas como mercancías (cuerpos) que no tienen un papel real en el cruce de fronteras (es trasladada, escondida), así la idea de su sufrimiento se materializa en una mirada lejana, objetivizada nuevamente. 
Debido al papel inofensivo asociado a las mujeres, ya sea por su naturaleza bondadosa o victimizada, incapaces de cometer delitos, las barreras fronterizas se consideran más fácilmente franqueables para ellas, por lo que son utilizadas como transporte. En el caso de las redes de tráfico de seres humanos y de prostitución, entre otros, son la mercancía que se intercambia entre fronteras. Las redes de tráfico de drogas emplean los cuerpos de las mujeres (aunque también el cuerpo de los hombres) como recipiente. Las "mujeres mulas" son utilizadas en Melilla para llevar mercancías de gran volumen a sus espaldas (que a veces incluso doblan su peso) de Marruecos a España. Las mujeres y sus cuerpos son instrumentos para el contrabando y para la comisión de actos delictivos (Enloe, 1989; Bloom, 2011; Cohn, 2015; Ponzanesi, 2014), porque, como afirma Sheila Jeffreys (2005) son igualmente objeto de protección y objeto de profanación. De esta manera, los cuerpos de las mujeres se convierten en medios para traspasar las fronteras de la legalidad, al mismo tiempo que son utilizados como moneda de cambio de los negocios de los hombres.

Si los cuerpos de las mujeres son objeto de la dominación patriarcal, incluso extralimitando los límites de las fronteras nacionales debido a la globalización, las tecnologías han facilitado la modulación de los gustos según la "disponibilidad de los cuerpos" femeninos (Meszaros, 2018). Lo que el enfoque de género aporta al análisis es la evidencia de que los cuerpos de los flujos migratorios son un elemento de consideración en sí mismo, puesto que es configurado por el modelo jerárquico de dominación patriarcal. Así, los cuerpos de hombres y mujeres están ligados a procesos identitarios y relacionales específicos, y reflejan experiencias y discursos diferentes de género. Mientras los cuerpos masculinos siguen siendo los conformadores de la fuerza laboral o el universal neutral, el de las mujeres siguen ocupando un rol pasivo (incluso si son mujeres terroristas son relacionalmente identificadas como "las esposas o las novias de los yihadistas"). 


\section{Conclusiones}

En el presente trabajo, se han examinado fronteras y la utilización de sistemas tecnológicos avanzados para controlar el flujo de población, tanto de la emigración como de la movilidad de los ciudadanos que gozan de derechos necesario para acceder a los países de destino. La tecnología, como tanto otros aparatos de poder de la sociedad, establece un protocolo de ordenación de los cuerpos, de las identidades y de los afectos (Foucault, 2002). Así, como el capitalismo ha establecido mecanismos de control sobre las mercancías, el liberalismo ha establecido mecanismos de control sobre nuestros derechos y capacidad de juicio crítico (Chun, 2006; Nussbaum, 2007; Levmore y Nussbaum, 2010). La plasticidad de los sentimientos de inseguridad y peligro se transfiere a nuestro sistema de valores, y se traslada a una funcionalidad fundamental de las tecnologías avanzadas, así como de las rutinas que la acompañan. Las tecnologías proporcionan la ilusión de espacio vigilado y controlado para la ciudadanía, alejando de sí la impresión de amenaza, la criminalidad, y la inseguridad terrorista representada por los otros. Sobre este eje, orbitan regulaciones y costumbres, hábitos adquiridos a partir de la implementación de sistemas tecnológicos que interfieren en nuestra cotidianidad, que han sido domesticadas por la ciudadanía global y viajera. La repetición de normas, asumidas como pautas protocolizadas, moldean nuestros comportamientos y disciplina nuestros cuerpos.

Este trabajo no pone en duda la necesidad de que existan dispositivos de control fronterizos, pero aborda críticamente las consecuencias de la intermediación de medidas tecnológicas para la de gestión de los flujos humanos. La adopción de protocolos de seguridad justificados por la amenaza y el miedo de la ciudanía con derechos facilita la capacidad de dejar en suspensión la agencia y la protección de los sujetos sin derechos. Como consecuencia de lo anterior, pone en riesgo los derechos tanto de las personas emigrantes como de la ciudadanía con derechos de movimiento. Las emociones son mercantilizadas 
para generar una aprobación completa y para crear una distinción más entre las personas con y sin derechos, y con derechos pero con distinta capacidad económica. Asistimos al imperio supraindividual de la tecnología, es decir, la consideración de que este es el recurso más eficiente con el que contamos para generar seguridad y bienestar. Esa ilusión facilita la cesión de nuestra confianza, agencia e incluso nuestra intimidad y principios. La invisibilidad de los dispositivos de control y el alejamiento respecto a aquellas otras personas que consideramos del otro lado de la frontera (también de nuestra realidad social), permite la aprobación incluso si se vulneran los derechos humanos. En este contexto, las agencias de seguridad y el mercado se afianzan en el control de las fronteras y en la distribución de derechos. Los actores privados proporcionan servicios particulares y exclusivos a quienes tienen capacidad económica para "crearse espacios protegidos" o a quienes pueden disponer de mayor bienestar.

Los espacios fronterizos se convierten en un lugar transitorio donde se ejerce el control y la segregación. Del otro lado, solo parece haber caos y deterioro de la convivencia. Se establece un paralelismo con la idea del lugar donde podríamos estar si no se fuera por esa vigilancia férrea de los dispositivos de seguridad. Las tecnologías electrónicas de control en las fronteras segregan a las personas, gestionando los derechos de quienes pueden entrar y quienes no pueden acceder, las mercancías que pueden ser transportadas y las personas viajeras que siguen las normas correctamente (en contraposición, quienes las infringen y amenazan la seguridad de todos los pasajeros). Hemos analizado cómo los protocolos de seguridad ignoran rasgos culturales particulares, pero también sospecha de personas racializadas o señaladas por características propias de un estatus "inferior". Hemos distinguido el impacto desigual que produce en los cuerpos de hombres y mujeres, ligados a roles adscritos por el sistema patriarcal, las tecnologías en las fronteras. 
La sociedad que queremos para el futuro requiere que adoptemos una posición reflexiva sobre las tecnologías utilizadas para controlar las fronteras. Merece que reflexionemos acerca de qué elementos están contribuyendo a generar mayor bienestar y respeto, para lo cual debemos adoptar una mirada atenta sobre el impacto que ejerce sobre nuestros derechos, cuerpos y mercancías. Necesita atender a los elementos negativos que nos hacen desprendernos de nuestros valores primarios, los derechos humanos, los derechos individuales de protección de la intimidad. De otra manera, asumimos una escasa capacidad de agencia, rutinas que reflejan comportamientos irreflexivos, decididos por un grupo de tecnócratas, tecnólogos o comercializadores. En su lugar, deberíamos adoptar un modelo de control negociado y aceptado por la ciudadanía, que en este momento tiene el conocimiento, la capacidad, y el derecho de defender y seguir respuestas responsables y éticas. 


\section{Bibliografía}

Abbing, Henriette. (2011). Age Determination of Unaccompanied Asylum Seeking Minors in the European Union: A Health Law Perspective. European Journal of Health Law 18(1):11-25.

Agger, Ben (2003). The virtual self: A contemporary sociology. London: Blackwell.

Ahmed, Sara (2004). Affective Economies. Social Text 79, 22(2): 117-139.

Ahmed, Sara (2010). The Promise of Happiness. Durham and London: Duke University Press.

Ajunwa, Ifeoma, Kate Crawford, y Jason Schultz (2017). Limitless Worker Surveillance. California Law Review 105(3): 734-76.

Akrich, Madeleine (2006). La description des objects techniques. En M. Akrich, Michel Callon y Bruno Latour Sociologie de la traduction: Textes fondateurs. Paris: Presses de l'École des Mines de Paris.

Alvites Baiadera, Angélica Paola (2019). Migraciones internacionales, fronteras y Estados. ¿Cómo interpretar el régimen de frontera desde América del Sur? Desafíos, 31(1): 123-156.

Beck, Ulrich (2008). La sociedad del riesgo: Hacia una nueva modernidad. Barcelona: Paidós.

Bijker, Wiebe E., Thomas P. Hughes, y Trevor J. Pinch (Eds.). (1987). The Social Construction of Technological Systems: New Directions in the Sociology and History of Technology. Cambridge: MIT Press.

Blázquez Graf, Norma, y Javier Flores (2005). Ciencia, Tecnología y Género en Iberoamérica. México: Universidad Autónoma de México.

Bloom, Mia (2011). Bombshell: Women and Terrorists. London: Hurst. 
Brown, Wendy (2015). Estados amurallados, soberanía en declive. Barcelona: Herder.

Castles,Stephen,y MarkJ.Miller(1993).The age of migration: International population movements in the modern world. Michigan University: Macmillan.

Crawford, Kate (2019) Artificial Intelligence's White Guy Problem. The New York Time, 25 Junio, <https://mww.nytimes.com/2016/06/26/opinion/ sunday/artificial-intelligences-white-guy-problem.html> [Consulta: 13 enero 2020]

Chun, Wendy H.K. (2006). Control and Freedom. Power and paranoia in the age of fiber optics. Cambridge M.A.: MIT Press.

Cohn, Carol (ed.) (2015). Las mujeres y las guerras. Barcelona: Bellaterra

Cooke, Philip, y Loet Leydesdorff (2006). Regional development in the knowledge-based economy: The construction of advantage. The journal of technology Transfer 31: 5-15.

Dean, Jodi (2007). Feminism, Communicative Capitalism, and the Inadequacies of Radical Democracy. En Dahlberg Lincoln y Eugenia Siapera Radical Democracy and the Internet. London: Palgrave Macmillan.

Ditton, Jason (2000). Crimen and the city. The British Journal of Criminology 40(4): 692-709.

D'Ignazio, Catheriney Klei, Lauren (2019). Teach Datalike an intersectional feminist! IT Press Open. Disponible en: https://mitpressonpubpub.mitpress. mit.edu/pub/c5vkehnm/release/5

Enloe, Cynthia (1989). Bananas, Beaches, Bases: Making Feminist Sense of International Politics. London: Pandora Press

Elster, Jon (2005). El cemento de la Sociedad. Las paradojas del orden social. Barcelona: Gedisa. 
Foucault, Michel (2002 [1975]). Vigilar y Castigar. Nacimiento de la prisión. Buenos Aires: Siglo XXI.

FRONTEX (2015). Best Practice Operational Guidelines for Automated Border Control (ABC) Systems. Warsaw: FRONTEX.

Goldsmith, Stephen, y Susan Crawford (2014). The Responsive City: Engaging Communities Through Data-Smart Governance. Chicago: JosseyBass, John Wiley \& Sons.

Grabitz, Eberhard (1992). Border Controls. En Rudolf Bernhardt Encyclopedia of Public International Law. Amsterdam: North-Holland.

Haraway, Donna (1988). Situated Knowledges: The Science Question in Feminism and the Privilege of Partial Perspective. Feminist Studies 14(3): 575-599.

Harding, Sandra (2004). The Feminist Standpoint Theory Reader: Intellectual and Political Controversies. London: Routledge.

Hughes, Thomas P. (1987). The Evolution of Large Technological Systems. En Wiebe E. Bijker, Thomas P. Hughes y Trevor J. Pinch (ed). The Social Construction of Technical Systems: New Directions in the Sociology and History of Technology. Cambridge, MA: MIT Press.

Institute for Economics \& Peace (2018). Global Terrorism Index 2018. Measuring the impact of terrorism. Sidney: Institute for Economics \& Peace. Disponible en: http://visionofhumanity.org/reports.

Jeffreys, Sheila (2005). Beauty and Misogyny: Harmful Cultural Practices in the West. London: Routledge.

Lessig, Lawrence (1999). Code Version: And Other Laws of Cyberspace. New York: Basic Books.

Levmore, Saul, y Martha C. Nussbaum (2010). The Offensive Internet. 
Privacy, Speech, and Reputation. Cambridge: Harvard University Press.

Lie, Merete y Sorensøn, Knut (eds.) (1996). Making technology our own? Domesticating technology into everyday life. Oslo: Scandinavian University Press.

Livingstone, Sonia, Mariya Stoilova, y Anthony Kelly (2016). Cyberbullying: incidence, trends and consequences. En UNITED NATIONS OFFICE Ending the Torment: Tackling Bullying from the Schoolyard to Cyberspace. New York: United Nations Office of the Special Representative of the SecretaryGeneral on Violence against Children.

Lunt, Peter, y Sonia Livingstone (2012). Media regulation: governance and the interests of citizens and consumers. London: SAGE.

Lyon, David (1994). Electronic Eye: The Rise of Surveillance Society. Oxford: Polity Press.

Meszaros, Julia (2018). Race, space, and agency in the international introduction industry: how American men perceive women's agency in Colombia, Ukraine and the Philippines. Gender, Place \& Culture 25:2: 268287.

Nussbaum, Martha (2007). Las fronteras de la justicia: consideraciones sobre la exclusión. Barcelona: Paidós.

Oroza, Rebeca, y Yoannis Puente Márquez (2017). Migración y comunicación:surelaciónenelactual mundoglobalizado.CEDEM/Novedades en Población 25: 10-16.

Ponzanesi, Sandra (Ed.) (2014). Gender, Globalization, and Violence Postcolonial Conflict Zones. New York: Routledge

Rodier, Claire (2010). Frontex, l'agence tout risqué. Plein droit 4(87): 8-11.

Sassen, Saskia (2007). Una sociología de la globalización. Buenos Aires y 
Madrid: Katz Barpal Editores.

Sassen, Saskia (2015). Expulsiones: brutalidad y complejidad en la economía global. Buenos Aires y Madrid: Katz Barpal Editores.

Thakor, Mitali, y danah boyd (2013). Networked trafficking: reflections on technology and the anti-trafficking movement. Dialect anthropology 37:277290.

Urry, John (2007). Mobilities. Cambridge: Polity Press.

Weiss, Linda (2014). America Inc.? Innovation and Enterprise in the National Security State. Cornell: Cornell University. 